\title{
Study on the Motion Characteristics of Residual Air Mass in Pipelines in Water Transfer Project
}

\author{
Huang Haocheng ${ }^{1}$, Jiang Jin ${ }^{1, a}$, Chen Qi $^{2}$, Liao Zhifang ${ }^{3}$, Liu Laiquan ${ }^{1}$ \\ ${ }^{1}$ Key Lab of Hydraulic Machinery Transient, MOE, Wuhan University, Wuhan, China \\ ${ }^{2}$ Hubei Water Resources Research Institute, Wuhan, China \\ ${ }^{3}$ Bensv Valve Stock co., Itd, Tianjin, China
}

\begin{abstract}
For long-distance water transfer projects, the residual air mass in the pipeline will not only reduce the efficiency, but also be detrimental to the safety of the system. In order to study the influence of the water flow velocity of the pressurized water pipeline and the pipeline angle of the hump on the motion characteristics of the residual air mass, an experimental platform with Particle Image Velocimetry (PIV) measuring system was constructed to analyse the flow field. The RSM turbulence model was combined with the VOF multiphase flow model to construct a local high-point gas-liquid two-phase fluid dynamics model for numerical simulation. The results showed that with the increase of water flow velocity, the local hump residual air mass would go through three states, namely, no bubble generation, air bubble was generated and partial discharged, and air mass discharge at one time. If the gas was greater than a certain volume, the increase in the water flow rate required to carry the air mass out of the hump at one time would slow down; the larger the local hump angle was, the greater the water flow rate was required to carry the gas out of the local hump part.
\end{abstract}

\section{Introduction}

\subsection{Background}

Long-distance water transfer pipeline projects are limited by terrain and construction conditions, and local humps will occur in pipeline layout. In the long-distance water supply pipeline, if the water delivery flow rate is small, the gas will stay in the local hump in the pipeline, the valve plate position in the pipeline, the reducer, the throttle Orifice plate, relatively flat reverse slope pipe section and other positions. It has been confirmed in a large number of projects that due to the change of slope, roughness and diameter of long-distance water pipelines, the air mass existing in the pipeline disperses and aggregates when flowing with water, which easily causes a drastic change in the pressure inside the pipeline.

When the pipeline system is running, the residual air mass will produce a pressure oscillation effect. On the one hand, it occupies the water passage section of the pipeline to reduce the pipeline operation efficiency, and on the other hand, there is a risk of water hammer accidents such as pipeline burst [1-2]. The literature [3-5] analysed the causes of accidents and economic losses caused by explosion accidents in actual projects, and proposed prevention and solutions.

In the water transfer project, air valves are usually installed at a local hump [6], which can effectively discharge the residual air mass at the local hump to ensure the safety of the water delivery system. For the local hump position of the pipeline without the air valve, it is an economical and feasible solution to control the water flow velocity of the pipeline so that the water flow will carry the residual air mass out of the local hump. Therefore, it is of great significance to study the motion characteristics of air mass in the pipeline to prevent water hammer damage.

\subsection{Application of PIV in two-phase flow}

PIV (Particle Image Velocimetry) technology has been widely used in hydrodynamic research as a method for measuring transient flow rate without contact. The basic principle of PIV is to input tracer particles with similar liquid phase density and good following ability in the flow field to describe the movement of the fluid at the corresponding position in the flow field in the motion of the tracer. The CCD (charge coupled device) camera is used to record successive moving images of tracer particles and analyse the captured continuous PIV images.

During the past decades, foreign and domestic scholars have carried out massive work on the application of PIV technology in multiphase flow. A Tokuhiro[7] et al. first conducted an experimental study on the attached oscillating bubble, using light-induced fluorescence (LIF) to measure the velocity of the bubble and the surrounding flow field, while recording the bubble shape and speed with a second CCD camera. M Birvalski[8] et al. experimentally studied the stratified wave currents of air

\footnotetext{
a Corresponding author: Jiang Jin, 00010482@whu.edu.cn
} 
and water in horizontal pipelines, simultaneously measured the velocity field in gas-liquid two-phase flow with PIV, and analyzed the shape of the interface using contour capture techniques in two waveform modes to study the interface characteristics and velocity of the liquid and gas phases. C Meyer[9] et al. measured the velocity distribution in the liquid film of the gas-liquid interface. Chen[10] et al. used PIV to observe the velocity distribution in Rayleigh convection caused by acetone volatilization of acetone-ethyl acetate binary system. Xu Ming[11] et al. developed a PIV experimental system for the study of oil-water two-phase flow, and measured the velocity field of the stratified flow and the dispersed flow in the horizontal oil-water two-phase flow respectively, which demonstrated a significant velocity slip between the phase and the water phase.

However, at present, there are few studies on the residual air mass in the water pipeline through PIV technology in China. In order to study the motion characteristics of the hump residual air mass in the pipeline to reduce the risk of safe operation of pipeline water conveyance caused by gas and improve the safety of pipeline operation, an experimental platform was built to observe and analyse the gas motion characteristics in the pipe section. The flow field at a local hump was measured with PIV system to study the effects of the water flow velocity in the pipeline, the volume of the retained air mass in the pipeline, and the downward angle of the hump pipeline on the motion characteristics of the retained air mass.

\section{Experiment and Numerical Simulation}

\subsection{Experimental devices}

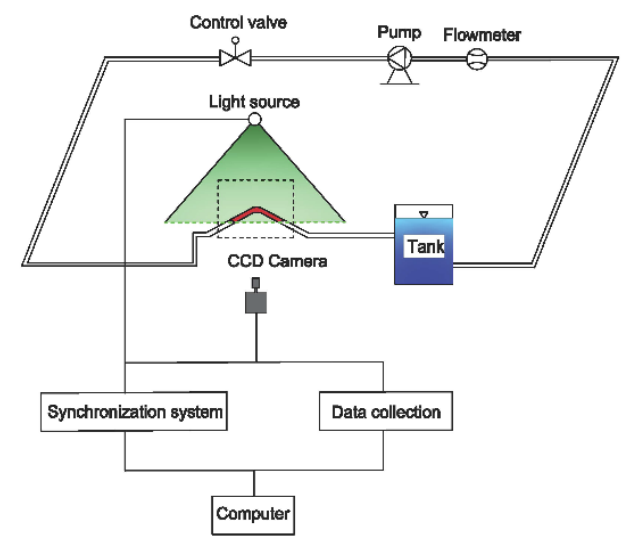

Fig.1 Experimental equipment

The device of this experiment consisted of three parts: hydraulic system components, measurement system and software analysis system. The hydraulic system was a circulating system consisting of a DN100 pipeline, a pump, a control valve, a transparent pipe section, and a tank. The measuring system included a PIV test system and a flowmeter. The experimental design conditions were rich enough to start a comprehensive study of the entire transient process performed.
The PIV model used in this experiment was V3V Flex from TSI, USA. The system included a light source, a CCD camera, a synchronization system and a data acquisition system to measure the flow field at a local hump $(150 \mathrm{~mm} \times 150 \mathrm{~mm})$. And the Insight V3V software was used to identify and calculate the displacement of the tracer particles in the image, and obtain the velocity vector and other motion parameters of each point in the flow field. The tracer particles selected in this study are hollow glass spheres with a density of $1.04 \mathrm{~g} / \mathrm{cm}^{3}$ and a particle diameter of $8-12 \mathrm{um}$, which can meet the experimental requirements of water as medium.

By adjusting the opening degree of the valve to increase the flow rate gradually, it could be preliminarily observed that the shape of the air mass would be experienced in three different states as the flow rate increases. The residual air mass at the local hump of the pipeline will experience three different states, namely: no air bubble generated at low speed; when the speed increased to a certain extent, bubbles would be generated at the tail of the air mass, and as the flow velocity increases, some of the air bubbles would be carried out to the hump; when the flow rate exceeded a certain number, the air mass would be carried out at the hump. In the water transfer pipeline project, the flow rate when being discharged at one time is more concerned. In order to determine the water flow velocity required for the air mass to be discharged at different angles and different residual air mass volumes, the following experiments were carried out:

1. The $10^{\circ}$ transparent hump tube was installed and 60 $\mathrm{cm}^{3}$ of air was injected into the transparent piece. Turned on the water pump and controlled the opening degree of the control valve to observe. When the air flow was taken away by the water flow once, the flow rate was recorded at this time;

2. The air with a volume of $260 \mathrm{~cm}^{3}, 300 \mathrm{~cm}^{3}, 460 \mathrm{~cm}^{3}$, $710 \mathrm{~cm}^{3}$ were injected separately and step 1 was repeated;

3. The $10^{\circ}$ transparent hump pipe fittings were removed and the transparent hump pipe fittings at $20^{\circ}$ and $30^{\circ}$ inclination angles were installed separately. Steps 1 and 2 were repeated to record the flow rate.

The above experiment was carried out to measure the flow rate when the air mass was once discharged into the pipeline at different angles or different air mass volumes, which was recorded as the critical flow rate.

As an example, a $20^{\circ}$ transparent hump pipe with a residual air mass of $260 \mathrm{~cm}^{3}$ was measured with PIV system to study the flow field in a transparent pipe at flow velocity of $0.2 \mathrm{~m} / \mathrm{s}, 0.5 \mathrm{~m} / \mathrm{s}$, and $0.8 \mathrm{~m} / \mathrm{s}$.

\subsection{Numerical Simulation}

The RSM turbulence model was combined with the VOF multiphase flow model to construct to analyse the flow field.

The VOF model was the fluid volume function model. For each grid in the flow field, the function was defined as the ratio of the volume of the target fluid to the volume of the grid. Solve the function values on each grid to track the motion interface. The RSM model,(Reynolds stress 
model), is a turbulence calculation model with high Re number.

$$
\begin{gathered}
\frac{\partial}{\partial t}\left(\alpha_{i} \rho_{i}\right)+\nabla \cdot\left(\alpha_{i} \rho_{i} v\right)=0 \\
\sum_{i=1}^{2} \alpha_{i}=1
\end{gathered}
$$

In the formula, the subscript $i$ represents the phase of the fluid in the model, $\rho$ is the density, and $v$ is the velocity.

The N-S equations are time-homogenized, according to the Reynolds stress term in the momentum equation of the Reynolds stress model, the continuity equation and the momentum equation are expressed by equations (2) and (3), respectively:

$$
\begin{gathered}
\frac{\partial \rho}{\partial t}+\frac{\partial}{\partial x_{i}}\left(\rho u_{i}\right)=0 \\
\frac{\partial}{\partial t}\left(\rho \overline{u_{i}^{\prime} u_{j}^{\prime}}\right)+\frac{\partial}{\partial x_{k}}\left(\rho u_{k} \overline{u_{i}^{\prime} u_{j}^{\prime}}\right)=\frac{\partial}{\partial x_{k}}\left[\mu \frac{\partial}{\partial x_{k}}\left(\overline{u_{i}^{\prime} u_{j}^{\prime}}\right)\right]- \\
\rho\left(\overline{u_{i}^{\prime} u_{k}^{\prime}} \frac{\partial u_{j}}{\partial x_{k}}+\overline{u_{j}^{\prime} u_{k}^{\prime}} \frac{\partial u_{i}}{\partial x_{k}}\right)+ \\
D_{T, i j}+G_{i j}+\phi_{i j}-\varepsilon_{i j}
\end{gathered}
$$

In the formula (3):

$$
\begin{aligned}
D_{T, i j}= & \frac{\partial}{\partial x_{k}}\left(\frac{\mu_{t}}{0.82} \frac{\partial \overline{u_{i}^{\prime} u_{j}^{\prime}}}{\partial x_{k}}\right) \\
G_{i j}= & -\rho \beta\left(g_{i} \overline{u_{j}^{\prime} \boldsymbol{\theta}}+g_{j} \overline{\left.u_{i}^{\prime} \boldsymbol{\theta}\right)}\right. \\
\phi_{i j}= & -1.8 \rho \omega \beta_{R S U}\left(\overline{u_{i}^{\prime} u_{j}^{\prime}}-\frac{2 \delta_{i j} k}{3}\right)- \\
& 8\left(P_{i j}-\frac{P_{k k} \delta_{i j}}{3}\right)- \\
& 0.2\left[-\rho\left(\overline{u_{i}^{\prime} u_{k}^{\prime}} \frac{\partial u_{k}}{\partial x_{j}}+\overline{u_{j}^{\prime} u_{k}^{\prime}} \frac{\partial u_{k}}{\partial x_{i}}\right)-\frac{P_{k k} \delta_{i j}}{3}\right]- \\
& 0.49 k\left(S_{i j}-\frac{S_{k k} \delta_{i j}}{3}\right) \\
\mathcal{E}_{i j}= & \frac{2}{3} \delta_{i j}\left(\rho \mathcal{E}+2 \rho \boldsymbol{\varepsilon} \frac{k}{a^{2}}\right)
\end{aligned}
$$

The Pro/E software was used to build the physical model of the local pipeline, and the ICEM software was used to generate the grid. When the mesh was generated, a more detailed trap sites were processed to obtain higher quality meshes. The inlet and outlet boundary conditions were set to $3 \times 10^{4} \mathrm{~Pa}$ and $5 \times 10^{4} \mathrm{~Pa}$, respectively.

The calculated time step is $0.001 \mathrm{~s}$ and the residual is set to $10^{-6}$. Four kinds of grids of $0.8 \times 10^{4}, 1.0 \times 10^{4}$, $2.3 \times 10^{4}$, and $3.5 \times 10^{4}$ were set. Through the independence analysis, the setting of the $1.0 \times 10^{4}$ grid number is adopted for calculation.

\section{Results and discussion}

\subsection{Critical flow rate analysis results}

The critical flow rate measured results are as follows: Table 1. The critical flow rate

\begin{tabular}{ccccc}
\hline & & $10^{\circ}$ & $20^{\circ}$ & $30^{\circ}$ \\
\cline { 2 - 4 } & $60 \mathrm{~cm}^{3}$ & 15.9 & 18.45 & 21.6 \\
\hline
\end{tabular}

\begin{tabular}{ccccc} 
& $260 \mathrm{~cm}^{3}$ & 18.65 & 21.13 & 24.7 \\
Flow rate & $330 \mathrm{~cm}^{3}$ & 20.01 & 21.86 & 25.6 \\
$\left(\mathrm{~m}^{3} / \mathrm{h}\right)$ & $460 \mathrm{~cm}^{3}$ & 20.02 & 22.43 & 27.4 \\
& $710 \mathrm{~cm}^{3}$ & 20.04 & 23.21 & 27.8 \\
\hline
\end{tabular}

Based on the measured critical flow rate, the critical flow velocity at which the air mass was carried out from the local hump were compared under various operating conditions.

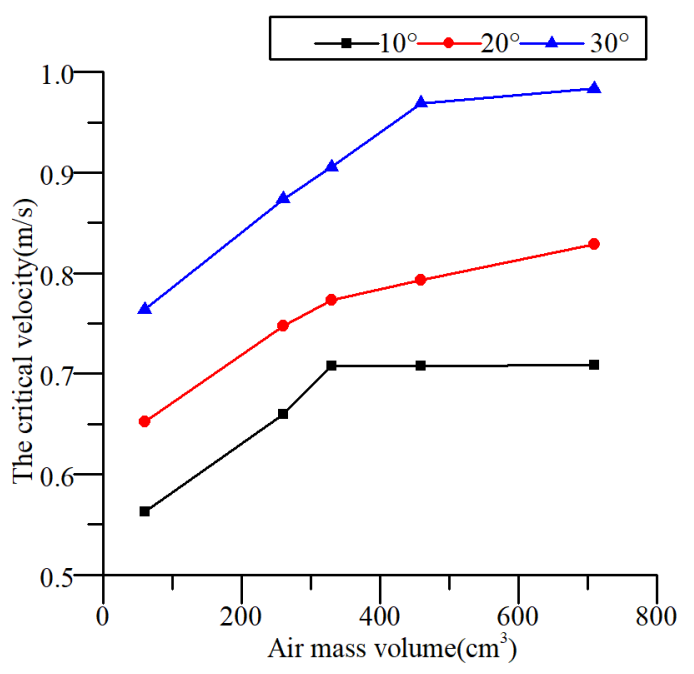

Fig.2 Curve of the critical flow velocity

The analysis was performed in comparison with Table 1 and Figure 2. By comparison, it could be seen that with the increase of the volume of retained air mass in the pipeline at the same angle, the critical flow velocity of the pipeline increases first, and the subsequent increase gradually decreases. The reason for this phenomenon is mainly because as the volume of the retained air mass increased, the impact force from the water flow received by the air mass needed to be able to overcome the buoyancy and the resistance of the pipe wall. Therefore, the larger the volume of the air mass, the greater the impact force was required to take it away at a time, so a larger flow rate was needed.

The results of experiments on pipes with different dip angles showed that the critical flow rate of the same volume of retained air mass would increase with an increase in angle. As the angle increases, the impact of the water flow on the air mass overcomes the buoyancy and the wall. The resistance increases, and the corresponding water flow rate required to carry the air mass out of the hump is larger.

\subsection{Flow field analysis}

In order to study the air mass characteristics at different flow rates, the PIV system and numerical simulation were used to analyse the air mass and liquid flow field in the transparent pipe at a flow velocity of $0.2 \mathrm{~m} / \mathrm{s}, 0.5 \mathrm{~m} / \mathrm{s}$ and $0.8 \mathrm{~m} / \mathrm{s}$, and liquid phase velocity distribution in the simulation calculation was obtained at $0.1 \mathrm{~s}, 1 \mathrm{~s}, 2 \mathrm{~s}, 3 \mathrm{~s}$. The captured measurement and numerical simulation results were as follows: 

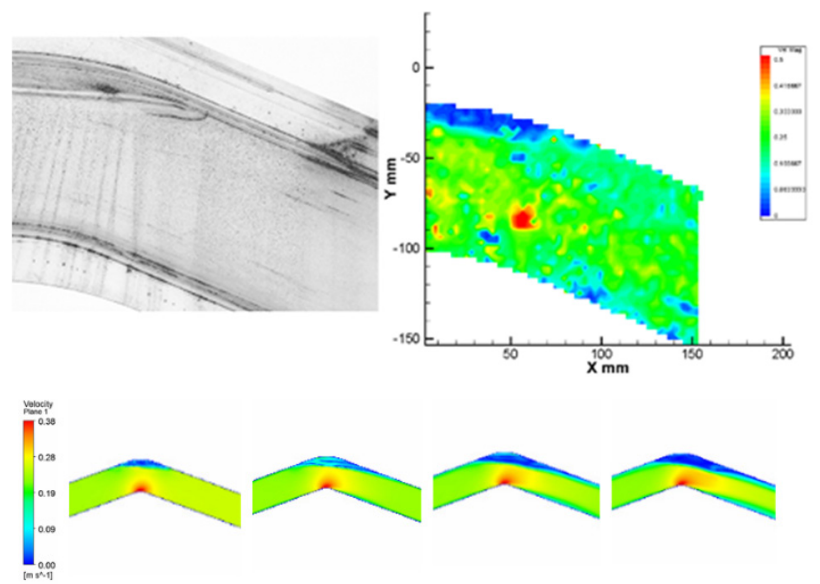

Fig.3 Velocity distribution at the flow velocity of $0.2 \mathrm{~m} / \mathrm{s}$

When the water velocity in the pipeline was $0.2 \mathrm{~m} / \mathrm{s}$, it could be observed from Fig. 3 that there was no significant morphological change in residual air mass, and the gasliquid interface was relatively stable with no obvious wave generation, and the turbulence at the local high point was not severe. The flow field measurement and simulation results showed that the flow velocity in the pipeline was relatively stable overall, but the velocity gradient was large in the radial direction at the gas-liquid interface.

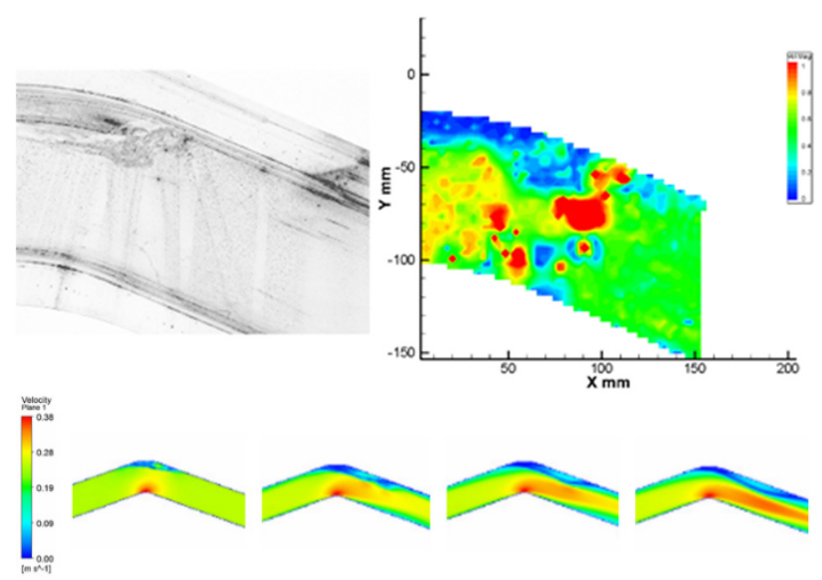

Fig.4 Velocity distribution at the flow velocity of $0.5 \mathrm{~m} / \mathrm{s}$

When the water flow velocity in the pipeline was 0.5 $\mathrm{m} / \mathrm{s}$, continuous bubbles were observed in the tail of the retained air mass as shown in Fig. 4. The gas-liquid interface of the pipeline fluctuated sharply, and the small enough bubbles were discharged as the water flows away. The large bubbles were combined with the large air mass due to the buoyancy and vortex. The flow field measurement and simulation results showed that the flow velocity inside the liquid phase at the tail of the air mass fluctuates greatly and the turbulence is severe. The interaction between the gas-liquid two-phase interface became larger and larger with time due to the impact of the water flow. In the actual experiment, the phenomenon was that there are continuous bubbles in the tail of the air mass.
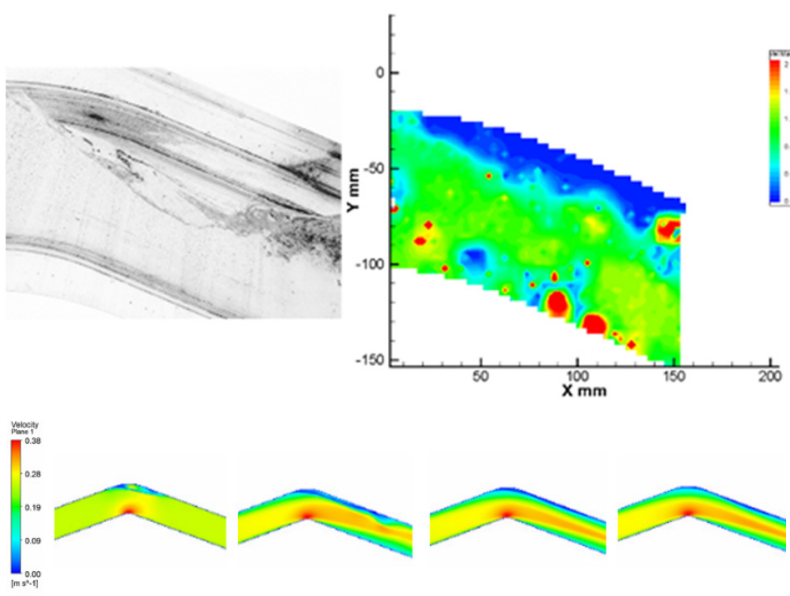

Fig.5 Velocity distribution at the flow velocity of $0.8 \mathrm{~m} / \mathrm{s}$

When the water flow velocity in the pipeline was 0.8 $\mathrm{m} / \mathrm{s}$, it could be found that the gas-liquid interface of the pipeline fluctuates drastically. At this time, the impact of the water flow on the air mass in the pipeline was sufficient for the air mass to overcome the buoyancy and resistance caused by the wall surface, so that a large amount of air bubbles were generated at the tail of the air mass, and the air mass was quickly carried along by the water flow. The flow velocity in the flow field fluctuated drastically. As the air mass was carried out, the flow velocity in the pipeline became relatively stable.

The comparison between the PIV experiment and the numerical simulation showed that the VOF and RSM combined model simulation could reflect the motion characteristics of residual air mass of the pipeline well.

\section{Conclusion}

In view of the problem of residual air mass in the pipeline in the actual water transfer project, the self-venting capacity of the pipeline in humps of a pipeline was studied. Through the experiment, analysis was made on the selfventing ability of pipelines under various conditions, and the following conclusions were obtained:

(1) There were three kinds of morphological changes with the increase of the flow velocity, that was, there was no significant change in the air mass at low speed; at the medium speed, bubbles would appear at the tail of the air mass, and the number of bubbles increased with the increase of the flow velocity; At a critical flow rate, the air mass would be carried along with the water flow.

(2) The critical flow velocity of the pipeline selfexhaustion was positively correlated with the angle and the volume of the air mass. As the volume of the air mass increased, the increase of the critical speed decreased.

(3) The numerical simulation method used in this study was in good agreement with the PIV measurement results, which could describe the flow characteristics of this experiment well.

This experiment was not perfectly carried out. In the experiment, the influence of pressure changes at both ends of the pipe on the volume and shape of the air mass was not considered, and only a circular cross-section pipe with 
the diameter of $100 \mathrm{~mm}$ was used. Further research can be conducted on the residual air mass in pipelines.

\section{References}

1. Wylie E B, Streeter V L. Fluid transients[M]. Osborne McGraw-Hill, 1978.

2. Yang Yusi, Zhang Shichang, Fu Lin. Harm and Protection of Airbag Movement in Pressure Water Supply Pipeline[J]. Chinese Water and Wastewater, 2002, 18(9): 32-33.

3. Romano M, Kapelan Z, Savić D A. Geostatistical techniques for approximate location of pipe burst events in water distribution systems[J]. Journal of Hydroinformatics, 2013, 15(3):634.

4. Wang R, Wang Z, Wang X, et al. Pipe Burst Risk State Assessment and Classification Based on Water Hammer Analysis for Water Supply Networks[J]. Journal of Water Resources Planning \& Management, 2013, 140(6):04014005.

5. Liu Zhiyong, Liu Meiqing, Jiang Jin, et al. Leakage detection of water pipeline based on transient flow frequency response analysis[J]. Journal of Hydraulic Engineering, 2015, 46(11): 1352-1359.

6. Jianyong, Zhang Jian, Ruan Ru. Study on Operation Characteristics of Air Valve in Long Distance Water
Transmission System[J]. Hydroelectric Power, 2007, 33(10): 61-63.

7. Tokuhiro A, Maekawa M, Iizuka K, et al. Turbulent flow past a bubble and an ellipsoid using shado image and PIV techniques[J]. International Journal of Multiphase Flow, 1998, 24(8):1383-1406.

8. Birvalski M, Tummers M J, Delfos R, et al. PIV measurements of waves and turbulence in stratified horizontal two-phase pipe flow[J]. International Journal of Multiphase Flow, 2014, 62(2):161-173.

9. Meyer C, Hoffmann M, Schlüter M. Micro-PIV analysis of gas-liquid Taylor flow in a vertical oriented square shaped fluidic channel[J]. International Journal of Multiphase Flow, 2014, 67:140-148.

10. Chen Wei, Chen Shuyong, Yuan Xigang, Zhang Huishu, Liu Botan, Yu Guozhen. PIV Experimental Observation of Rayleigh Convection and Its Influence on Mass Transfer[J].Journal by Chemical Engineering,2014,22(10):1078 -1086.

11. $\mathrm{Xu}$ Ming, Ling Hangjian, Wang Luhai, et al. Application of PIV Technology in Near-Level OilWater Two-Phase Flow Research[J]. Journal of Experimental Fluid Mechanics, 2012, 26(1): 12-15. 